\title{
Spatiotemporal modelling of ozone distribution in the State of California
}

\author{
P. Bogaert ${ }^{\mathrm{a}}$, G. Christakos $^{\mathrm{b}}$, M. Jerrett ${ }^{\mathrm{c}}$, H.-L. Yu ${ }^{\mathrm{d}, *}$ \\ ${ }^{a}$ Dept. of Environmental Sciences É Land Use Planning, Université Catholique de Louvain, Louvain-la-Neuve, Belgium \\ ${ }^{\mathrm{b}}$ Dept. of Geography, San Diego State University, San Diego CA, USA \\ ${ }^{\mathrm{c}}$ Dept. of Environmental Health Sciences, School of Public Health, University of California, Berkeley, CA, USA \\ d Dept. of Bioenvironmental Systems Engineering, National Taiwan University, No. 1 Roosevelt Rd., Sec. 4, Taipei 10617, Taiwan
}

\section{A R T I C L E I N F O}

\section{Article history:}

Received 30 September 2008

Received in revised form

24 December 2008

Accepted 28 January 2009

\section{Keywords:}

Air pollution

Ozone

California

Random fields

BME

Seasonal variations

GIS

Mapping

\begin{abstract}
A B S T R A C T
This paper is concerned with the spatiotemporal mapping of monthly 8-h average ozone $\left(\mathrm{O}_{3}\right)$ concentrations over California during a 15-years period. The basic methodology of our analysis is based on the spatiotemporal random field (S/TRF) theory. We use a S/TRF decomposition model with a dominant seasonal $\mathrm{O}_{3}$ component that may change significantly from site to site. $\mathrm{O}_{3}$ seasonal patterns are estimated and separated from stochastic fluctuations. By means of Bayesian Maximum Entropy (BME) analysis, physically meaningful and sufficiently detailed space-time maps of the seasonal $\mathrm{O}_{3}$ patterns are generated across space and time. During the summer and winter months the seasonal $\mathrm{O}_{3}$ concentration maps exhibit clear and progressively changing geographical patterns over time, suggesting the existence of relationships in accordance with the typical physiographic and climatologic features of California. BME mapping accuracy can be superior to that of other techniques commonly used by EPA; its framework can rigorously assimilate useful data sources that were previously unaccounted for; the generated maps offer valuable assessments of the spatiotemporal $\mathrm{O}_{3}$ patterns that can be helpful in the identification of physical mechanisms and their interrelations, the design of human exposure and population health models, and in risk assessment. As they focus on the seasonal patterns, the maps are not contingent on short-time and locally prevalent weather conditions, which are of no interest in a global and nonforecasting framework. Moreover, the maps offer valuable insight about the space-time $\mathrm{O}_{3}$ concentration patterns and are, thus, helpful for disentangling the influence of explanatory factors or even for identifying some influential ones that could have been otherwise overlooked.
\end{abstract}

(c) 2009 Elsevier Ltd. All rights reserved.

\section{Introduction}

Tropospheric ozone $\left(\mathrm{O}_{3}\right)$ is photochemically produced from the combination of volatile organic compounds and oxides of nitrogen in the presence of sunlight (Clark, 1980; Trainer et al., 1987; Logan, 1989; Diem, 2000). Remarkably, due to increased anthropogenic emissions of nitrogen oxides, background levels of $\mathrm{O}_{3}$ have doubled since the mid-19th century (Finlayson-Pitts and Pitts, 1997). Increases in $\mathrm{O}_{3}$ are a public health concern because the gas can enter the lung and exert oxidative stress (Carroll et al., 1997; Mudway and Kelly, 2004; Koike and Kobayashi, 2004). As a matter of fact, numerous research studies have linked $\mathrm{O}_{3}$ to cardiorespiratory health problems in humans (Chen et al., 2004, 2007). The dynamic distribution of $\mathrm{O}_{3}$ in various parts of the world has been studied using spatiotemporal statistics and integrated emission models (Christakos and Vyas, 1998; Christakos and Kolovos, 1999;

\footnotetext{
* Corresponding author. Tel.: +8862 33663454; fax: +886223635854.

E-mail address: hlyu@ntu.edu.tw (H.-L. Yu).
}

Tao et al., 2005; Christakos et al., 2004; Yu et al., 2008). Questions about $\mathrm{O}_{3}$ health hazards correspond to three basic lines of investigation (Christakos and Hristopulos, 1998): (a) define the exposure conditions across space-time; $(b)$ relate exposure to burden in the human body; and $(c)$ detect adverse health effects and assess population damages.

Exposure generally refers to the contact of a human receptor with the environmental pollutant. In observational epidemiology, usually exposure is considered equal to the airborne pollutant concentration at a specific point in space and time in relation to the human population receptor (Schneider et al., 1997; Thurston and Ito, 2001; Sram et al., 2005). As a result, the generation of realistic spatiotemporal $\mathrm{O}_{3}$ maps is essential in the evaluation of the assumptions underlying the health response models and the assessment of the damage on human populations due to $\mathrm{O}_{3}$ exposure (Solomon et al., 2000; Tao et al., 2004).

The present study is part of a multiyear investigation into the effects of ambient air pollutant exposure on mortality in California. The study builds on earlier research (Jerrett et al., 2005) with the specific aim of improving the spatiotemporal exposure assessment 
in order to rigorously estimate the mortality effects in a multipollutant context.

\section{Spatiotemporal statistics model}

Spatiotemporal analysis based on the Bayesian Maximum Entropy (BME) theory (Christakos, 1992, 2000; Christakos et al., 2002) distinguishes between: ( $a$ ) the core (or general) knowledge base (KB), denoted by $G-K B$ (it may include physical laws, primitive equations, and theoretical models of space-time dependence); and (b) the specificatory (or site-specific) KB, S-KB, which may include exact numerical values across space (hard data), intervals (there is not a unique data value available at a location but, instead, an interval of possible values), and probability functions (the datum at the specified space-time location has the form of a probability distribution). The total $\mathrm{KB}$ is denoted by $K=G \cup S$, i.e. it includes both the core and the site-specific KB.

Due to the considerable physical uncertainty (caused by physiographic characteristics, emission fluctuations, meteorological conditions, etc.), the exposure (e.g., the $\mathrm{O}_{3}$ exposure distribution) is represented as a spatiotemporal random field $X_{p}$ (S/TRF; Christakos and Hristopulos, 1998). The S/TRF domain is denoted by $\boldsymbol{p}=(\boldsymbol{s}, t)$, in which $s$ refers to spatial coordinates and $t$ is time. Specification of the attribute values at all points in a space-time domain specifies a S/TRF realization. Randomness manifests itself as an ensemble of possible realizations regarding the $\mathrm{O}_{3}$ distribution.

In light of the above considerations, the fundamental BME equations of spatiotemporal analysis are as follows (Christakos, 2008)

$$
\left.\begin{array}{l}
\int d \boldsymbol{\chi}(\boldsymbol{g}-\overline{\mathbf{g}}) \mathrm{e}^{\mu^{T} g}=0 \\
\int d \chi \xi_{S} \mathrm{e}^{\mu^{T} g}-A f_{K}(\boldsymbol{p})=0
\end{array}\right\},
$$

where $\mathbf{g}$ is a vector of $g_{\alpha}$-functions $(\alpha=1,2, \ldots)$ that represents stochastically the $G-\mathrm{KB}$ under consideration (the bar denotes statistical expectation), $\boldsymbol{\mu}$ is a vector of $\boldsymbol{\mu}_{\alpha}$-coefficients that depends on the space-time coordinates and is associated with $\mathbf{g}$ (i.e., the $\boldsymbol{\mu}_{\alpha}$ express the relative significance of each $g_{\alpha}$-function in the composite solution sought), the $\xi_{S}$ represents the $S-\mathrm{KB}$ available, $A$ is a normalization parameter, and $f_{K}$ is the attribute probability density function (pdf) at each point (as before, the subscript $K=G \cup S$ means that $f_{K}$ is based on the blending of the $G$ - and $S$ $\mathrm{KB})$. The $\mathbf{g}$ and $\xi_{S}$ are inputs to Eq. (1), whereas the unknowns are the $\mu$ and $f_{K}$ across space and time.

The $G-K B$ refers to the entire $\boldsymbol{p}$-domain of interest, which consists of the space-time point vector $\boldsymbol{p}_{k}$ where exposure estimates are sought and the vector $\boldsymbol{p}_{\text {data }}$ where site-specific information is available. The $G-\mathrm{KB}$ may include theoretical space-time dependence models of the attribute $X_{\boldsymbol{p}}$ (mean, covariance, variogram, generalized covariance, multiple-point statistics, and heterogeneity orders). Mean and covariance (variogram) are most commonly used in spatiotemporal analysis (Huang et al., 2007).

For practical purposes, the data point vector $\boldsymbol{p}_{\text {data }}$ consists of the hard data vector $\boldsymbol{p}_{\text {hard }}$ (where exact $\mathrm{O}_{3}$ measurements exist) and the soft data vector $\boldsymbol{p}_{\text {soft }}$ (where incomplete yet valuable $\mathrm{O}_{3}$ information is available; Taylor, 1993). For illustration, let exact measurements be available at $\boldsymbol{p}_{\text {hard }}=\left(\boldsymbol{p}_{1}, \ldots, \boldsymbol{p}_{32}\right)$, i.e., $X_{\boldsymbol{p} 1}=5.1, \ldots, X_{\boldsymbol{p} 32}=9.3$ (in proper units); and uncertain $O_{3}$ data of the form $3.2<X_{p 33}<4.1, \ldots$, $5.2<X_{\boldsymbol{p} 87}<6.4$ exist at $\boldsymbol{p}_{\text {soft }}=\left(\boldsymbol{p}_{33}, \ldots, \boldsymbol{p}_{87}\right)$. This sort of site-specific information is mathematically expressed by $\operatorname{Prob}\left[X_{p 1}=5.1, \ldots\right.$, $\left.X_{\boldsymbol{p} 32}=9.3\right]=P_{S}\left[X_{\boldsymbol{p} 1}=5.1, \ldots, X_{\boldsymbol{p} 32}=9.3\right]=1$ and $P_{S}\left[3.2<X_{\boldsymbol{p} 33}<4.1\right.$, $\left.\ldots, 5.2<X_{\boldsymbol{p} 87}<6.4\right]=1$, respectively. Now assume that at $\boldsymbol{p}_{24}$ the uncertain datum is expressed by the pdf $f_{S}\left(\boldsymbol{p}_{24}\right)$; then, $P_{S}\left[X_{\boldsymbol{p}_{24}}<\chi\right]=\int_{-\infty}^{\chi} \mathrm{d} \chi f_{S}\left(\boldsymbol{p}_{24}\right)$.

Theoretical studies have shown that the spatiotemporal BME analysis has a number of attractive features:
- It makes no restrictive assumptions concerning the linearity and normality of the exposure estimator (nonlinear estimators and non-Gaussian laws are automatically incorporated).

- It can study exposure with heterogeneous space-time dependence patterns and can synthesize various kinds of knowledge bases (core and site-specific) in a rigorous and general framework rather than in an ad hoc and artificial manner.

- It provides a sound space-time exposure characterization in terms of the complete pdf at every estimation point rather than just the first two estimation moments. Indeed, model $f_{K}$ in Eq. (1) describes the complete probability distribution of exposure values at each estimation point $\boldsymbol{p}_{k}$ in view of the total knowledge, $K-\mathrm{KB}$. In this way, more than one possibility can be considered at each point, as far as exposure estimation is concerned. E.g., given $f_{K}$ at $\boldsymbol{p}_{k}$, different $O_{3}$ exposure estimates can be derived at each node of the mapping grid (most probable, error minimizing, etc.), depending on the objectives of the study.

- It can readily consider uncertain yet valuable information at the exposure estimation points themselves, when available.

- It derives certain mainstream techniques (e.g., statistical regression and kriging) as its special cases, thus demonstrating BME's generalization power. E.g., when the KB is limited to hard data and 2nd-order space-time statistics, the BME obtains simple kriging as a special case (Christakos, 2000).

In this work, the spatiotemporal BME analysis was implemented using the publicly available SEKS-GUI software library (Kolovos et al., 2006; Yu et al., 2007).

\section{The ozone dataset}

The $\mathrm{O}_{3}$ levels are high enough in the state of California during the summer period to cause significant concern (Ostro et al., 2006). The dataset used in this study consisted of monthly $\mathrm{O}_{3}$ measurements (in ppm) for the period January 1988-December 2002 at the 262 sites state-wise monitored by federal, state and local governments and amalgamated into a unified dataset by the California Air Resources Board (Fig. 1).

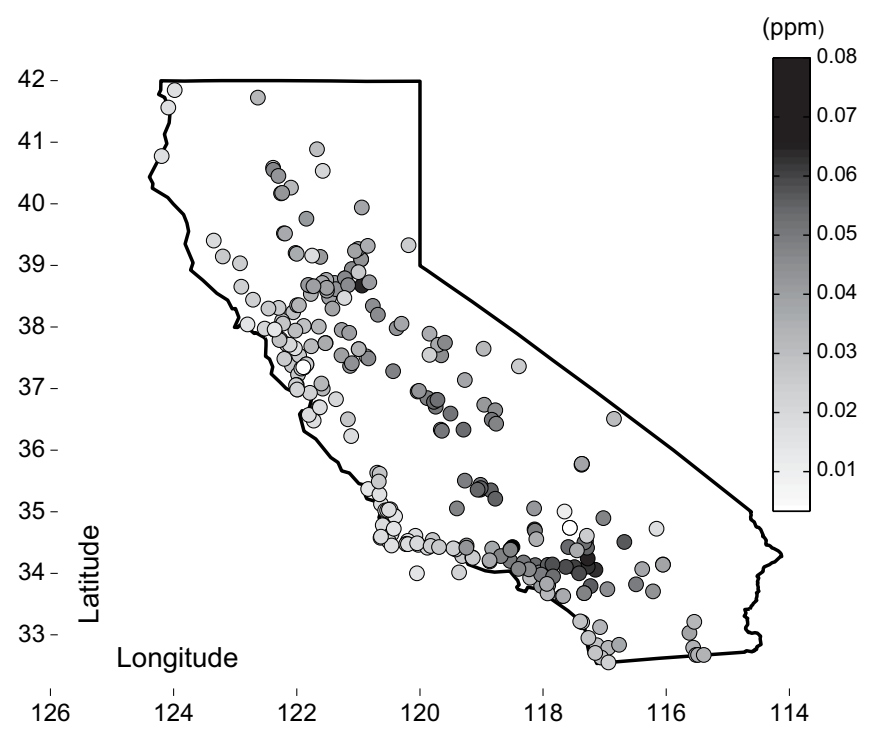

Fig. 1. Locations of the 262 sites that were active (i.e., at least one measurement was available) for 8-h average monthly $\mathrm{O}_{3}$ monitoring during the years 1988-2002, along with the amplitude (in $\mathrm{ppm}$ ) of the estimated seasonal component (lowest amplitude in black and highest amplitude in white). 
Measurements are monthly estimates based on 8-h $\mathrm{O}_{3}$ averages. Although this space-time sampling design would potentially include a dataset of 180 months $\times 262$ sites $=47,160$ data values, the 262 time-series have about $42 \%$ missing values. Part of the missing $\mathrm{O}_{3}$ values is associated with sites where $\mathrm{O}_{3}$ monitoring started or stopped during the years 1988-2002. Other missing values are associated with temporary failures or with stations monitored on a non-systematic temporal basis.

\subsection{Spatial and temporal features}

As is shown in Fig. 1, the monitoring sites tend to be clustered over space, roughly in line with major population centers. Although they yield a satisfactory coverage of the coastal area (except, perhaps, of the very northern part of California), this is not the case for the central part of the state. The North and East state borders are almost not covered at all. Fig. 2 shows that there is a clear seasonal effect, with $\mathrm{O}_{3}$ concentrations typically peaking during JulyAugust, and reaching their lowest values during December-January (as was expected on the basis of the physical and chemical processes of $\mathrm{O}_{3}$ generation, dispersion and disappearance; Chang et al., 1987; Jacobson, 1999).

It is worth noting that the amplitude and the shape of this seasonal $\mathrm{O}_{3}$ pattern may greatly vary from one station to another; e.g., the seasonal pattern exhibits sharp maxima at some sites, but it is constant or even not clearly observable at other sites. Despite these dissimilarities, there is no indication of a systematic increase or decrease of $\mathrm{O}_{3}$ concentrations during the 15 years covered by the dataset.

\subsection{Preliminary statistics}

According to the previous observations, and in order to assess the global contribution of the seasonal $\mathrm{O}_{3}$ pattern, an analysis of
Table 1

The $\mathrm{O}_{3}$ fluctuation variance of the three models in Eq. (2).

\begin{tabular}{llrlc}
\hline Model & $n$ & $p$ & SSE $/ n-p$ & $\% \mathrm{Var}$ \\
\hline Eq. (2a) & 27,444 & 1 & $3.09 \mathrm{e} 4$ & 0 \\
Eq. (2b) & 27,444 & 262 & $2.18 \mathrm{e} 4$ & 29 \\
Eq. (2c) & 27,264 & 2808 & $4.17 \mathrm{e} 5$ & 86 \\
\hline
\end{tabular}

Note: Above, $n$ is the number of data used in the model; $p$ is to the number of model parameters; $\operatorname{SSE} / n-p$ refers to the estimated variance of the fluctuations $\varepsilon_{\boldsymbol{p}}$; variance for model (2a) is the total variance, equal to $3.09 \mathrm{e} 4 \mathrm{ppm}^{2} ; \%$ Var refers to the $\%$ of explained variance by the model compared to model (2a).

variance (ANOVA) was conducted that involved the entire dataset. Let us consider the three following models

$X_{\boldsymbol{p}}=m+\varepsilon_{\boldsymbol{p}}$

$X_{\boldsymbol{p}}=m_{\boldsymbol{s}}+\varepsilon_{\boldsymbol{p}}$

$X_{\boldsymbol{p}}=m_{\boldsymbol{s} k}+\varepsilon_{\boldsymbol{p}} \quad$ (c)

where $\varepsilon_{\boldsymbol{p}}$ is a fluctuation field with $\overline{\varepsilon_{\boldsymbol{p}}}=0$ and $k=t(\bmod 12)$; $\bmod$ denotes the modulo operator. Model (2a) assumes the same mean $m$ at all sites and time instants; model (2b) assumes a different mean $m_{\boldsymbol{s}}$ at each site $\boldsymbol{s}$; and model (2c) assumes a different mean $m_{\boldsymbol{s} k}$ at each site $\boldsymbol{s}$ and each month $t$ (i.e., there are 12 possible values of the mean at each site, since the seasonal pattern exhibits an 1year period). Table 1 shows the variances $\operatorname{Var}[\varepsilon(\cdot)]$ of the fluctuations in Eq. (2). Both $n$ and $p$ in Table 1 may change with the model (2), since some of the $p$ parameters might be non estimable due to missing values. For our spatiotemporal design, if there were no missing values, $n$ would be equal to $180 \times 262=47,160$ for all models, whereas $p$ would be equal to 1262 and $12 \times 262=3144$ for models (2a)-(2c), respectively.

No classical statistics testing applies in this case (e.g., since the data are physically correlated across space-time, the $\varepsilon_{\boldsymbol{p}}$-independence hypothesis required by classical statistics does not hold true;
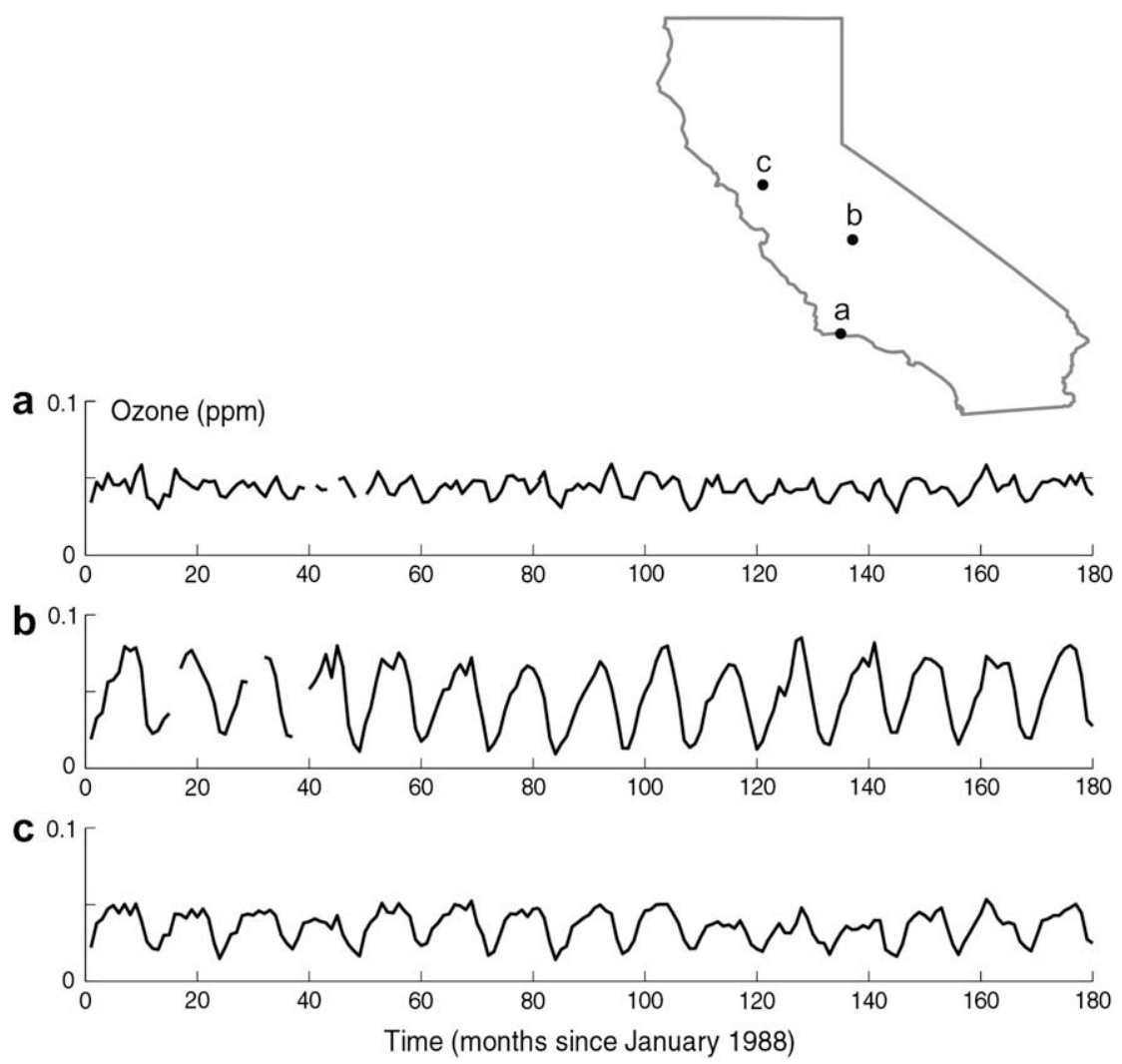

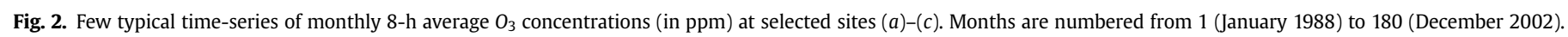


e.g., Mutshinda et al., 2007). There is, however, little doubt that, on the average, most of the observed variability is linked to the seasonal pattern. The estimated $\varepsilon_{\boldsymbol{p}}$-variance in model (2c) is about $14 \%$ of the total variance, so $\mathrm{O}_{3}$ fluctuation modelling is expected to play a rather minor influence on the final result, compared to the modelling of the seasonal effect.

\section{An ozone decomposition model}

According to the previous findings, let us now assume that the $\mathrm{S} /$ TRF $X_{\boldsymbol{p}}$ representing the $\mathrm{O}_{3}$ distribution can be decomposed as follows,

$X_{\boldsymbol{p}}=S_{\boldsymbol{p}}+\varepsilon_{\boldsymbol{p}}$ where $S_{\boldsymbol{p}}$ is a cyclic seasonal component with $\overline{S_{\boldsymbol{p}}}=m_{\boldsymbol{s}}$ (i.e., $\mathrm{O}_{3}$ concentration at location $s$ cyclically fluctuate through time around a time-independent value $m_{\boldsymbol{s}}$ ) and $\varepsilon_{\boldsymbol{p}}$ is the fluctuation component with $\overline{\varepsilon_{\boldsymbol{p}}}=0$. Assuming that $S_{\boldsymbol{p}} \perp \varepsilon_{\boldsymbol{p}}$ (the symbol " $\perp$ " denotes stochastic independence), the above belongs to the class of additive space-time models (Bogaert and Christakos, 1997a,b). From Eq. (3), the following properties hold true,

$$
\begin{aligned}
& S(\boldsymbol{s}, t)=S(\boldsymbol{s}, t+12) \\
& \overline{S(\boldsymbol{s}, t)}=\frac{1}{12} \sum_{i=1}^{12} S(\mathbf{s}, t+i)=m_{\mathbf{s}} \quad \text { (b) }
\end{aligned}
$$

In view of Eq. (4a), the symbols $S_{\boldsymbol{p}}=S(\boldsymbol{s}, t)$ and $S(\boldsymbol{s}, k)$ can be used equivalently to denote the seasonal component, where $k=t(\bmod$
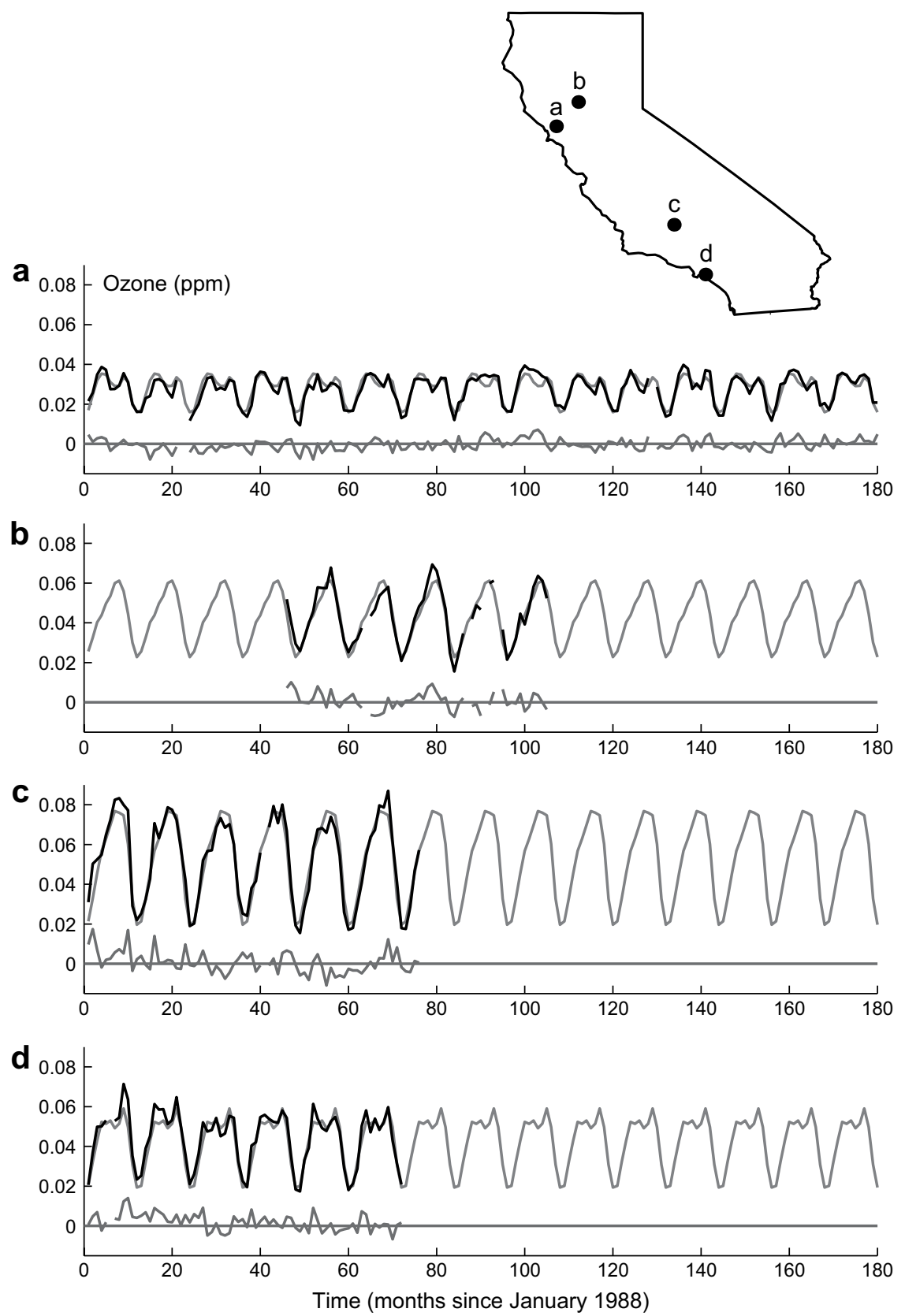

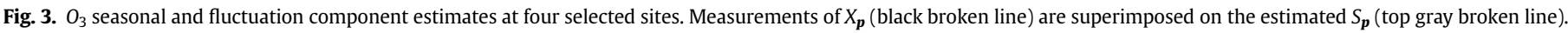
Estimates of the fluctuations $\varepsilon_{\boldsymbol{p}}$ (bottom gray line) were obtained by differencing at times where $X_{\boldsymbol{p}}$ values were available. 
12). Eqs. (3) and (4) correspond to Eq. (2c) with a set of twelve $S(\boldsymbol{s}, k)$ per site (i.e., 1 every month of the year).

As we saw in Section 2 above, the $G-K B$ may include theoretical spatiotemporal dependence models of the $\mathrm{O}_{3}$ distribution, such as the covariance. Accordingly, on the basis of the analysis in Appendix A, a valid spatiotemporal covariance model for $\varepsilon_{\boldsymbol{p}}$ is

$C_{\varepsilon}(\boldsymbol{h}, \tau)=\sigma_{\varepsilon}^{2}\left[a_{0} \delta_{0}+\left(1-a_{0}\right) \mathrm{e}^{-3 \boldsymbol{h} / a_{1}}\right] \mathrm{e}^{-3 \tau / a_{2}}$

with $\delta_{0}=\delta(\boldsymbol{h}=\mathbf{0}), \sigma_{\varepsilon}^{2}=2.75 \mathrm{e}-5 \mathrm{ppm}^{2}, a_{0}=0.48, a_{1}=1 e 5 \mathrm{~m}$ and $a_{2}=4$ months. The separable model (5) will be used in $\mathrm{O}_{3}$ modelling and mapping to represent spatiotemporal dependence of the fluctuation component.

Due to periodicity of the seasonal $\mathrm{O}_{3}$ component $S_{\boldsymbol{p}}$, it cannot be adequately modelled as a temporal random process (at a given location), but it can still be modelled as a spatial random field. It is, thus, possible to obtain its spatiotemporal covariance function $C_{S}(\boldsymbol{h}$, $k$ ), where $k$ denotes the month of the year considered. On the basis of the analysis in Appendix C, a valid spatiotemporal covariance model for $S_{\boldsymbol{p}}$ is given by

$C_{S}(\boldsymbol{h}, k)=\sigma_{S}^{2}(k) \mathrm{e}^{-3 \boldsymbol{h} / a}$

where $a=2 e 5 \mathrm{~m}$ and the $\sigma_{S}^{2}(k)$ values for each month $k$ are given in Fig. C.2b.

\section{Spatiotemporal ozone mapping}

Since the space-time component $\varepsilon_{\boldsymbol{p}}$ accounts only for a small fraction of the total $\mathrm{O}_{3}$ variability and exhibits low-level spatiotemporal correlations, its estimation is of limited interest in a global context. This means that spatiotemporal mapping can focus on the seasonal component $S_{\boldsymbol{p}}$ and that only 12 maps need to be drawn, as this component is assumed to repeat itself over the years. Next, spatiotemporal $\mathrm{O}_{3}$ maps were generated using the SEKS-GUI software library.

Estimating the seasonal component across space and time would require observing a certain set of $S_{\boldsymbol{p}}$ values. Since $S_{\boldsymbol{p}}$ was well approximated by $\widehat{S}_{\boldsymbol{p}}$ for the set of months and locations in which the condition $n(s, k) \geq 10$ is satisfied (Appendix C), using only this set of $S_{\boldsymbol{p}}$ values would leave out data from the 162 other sites that were discarded because they did not meet this condition. Additionally, as seen in Fig. C.1, this would also imply a quite poor spatial coverage for $\mathrm{O}_{3}$ mapping purposes in the state of California, since the 100 retained sites are mainly located along the coast, with none of them in the Eastern part of the state and very few of them in the central part of it.

Nevertheless, imposing a minimum value for $n(\cdot)$ was only required for the reliable estimation of $C_{S}(\boldsymbol{h}, k)$, but in a spatiotemporal mapping context all $\widehat{S}_{\boldsymbol{p}}$ obtained from Eq. (C.1) can be considered as soft data $(S-\mathrm{KB})$ of the true but unknown $S_{\boldsymbol{p}}=S(\boldsymbol{s}, k)$. Interestingly, in the context of the BME formalism these soft data can be properly assimilated in the efficient mapping of the seasonal $\mathrm{O}_{3}$ component. In particular, this includes spatiotemporal mapping of $S_{\boldsymbol{p}}$ at:

(a) non sampled (off-site) locations during various times (i.e., the classical mapping framework);

(b) sites where measurements are missing during certain times (i.e., typical estimation at one of the 162 discarded sites); and

(c) sites and times where soft data $\widehat{S}(\boldsymbol{s}, k)$ are already available.

Although all three cases are covered by the same mathematical BME formalism, they have different objectives and they address
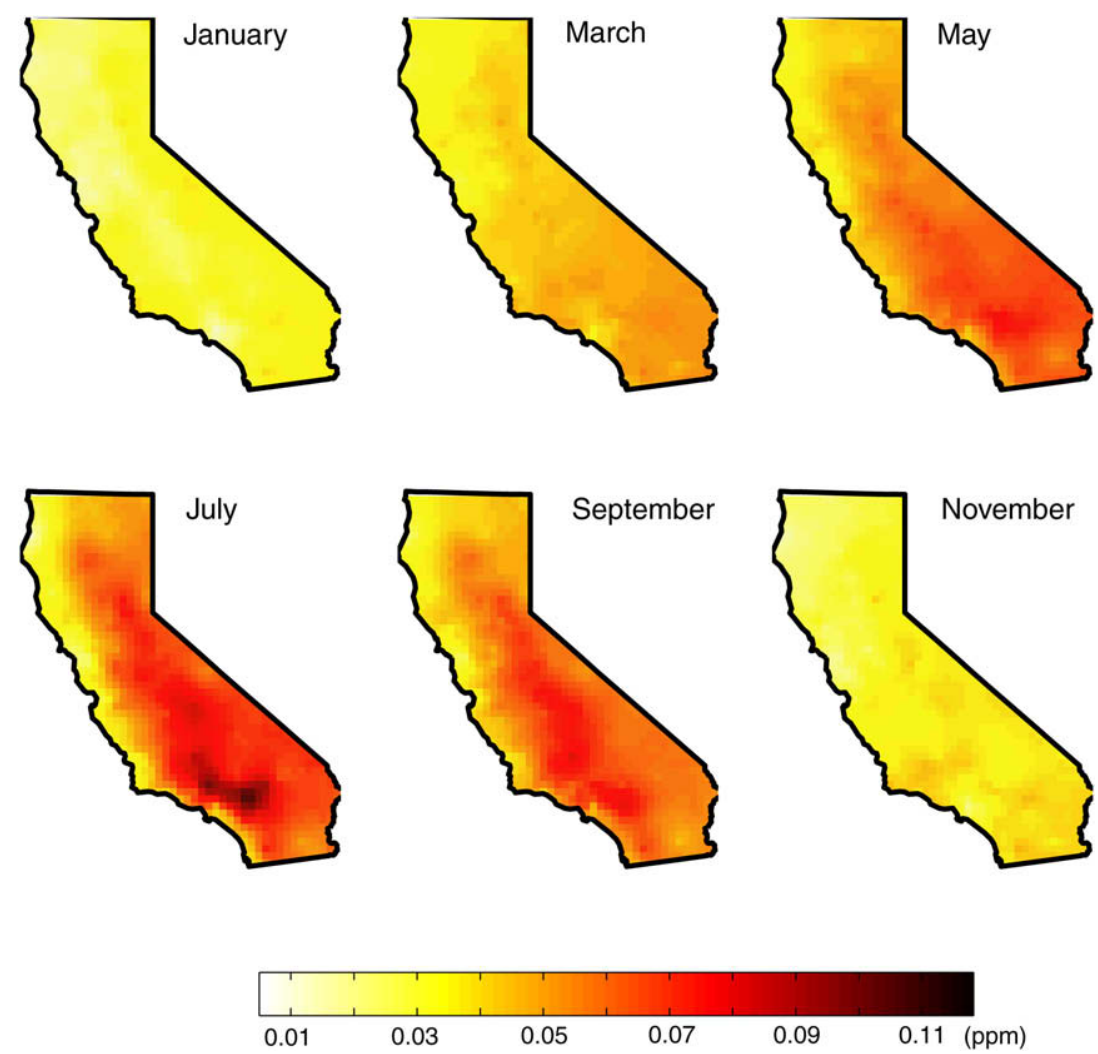

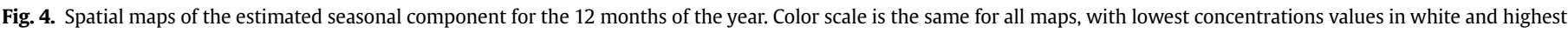
concentration values in black. 

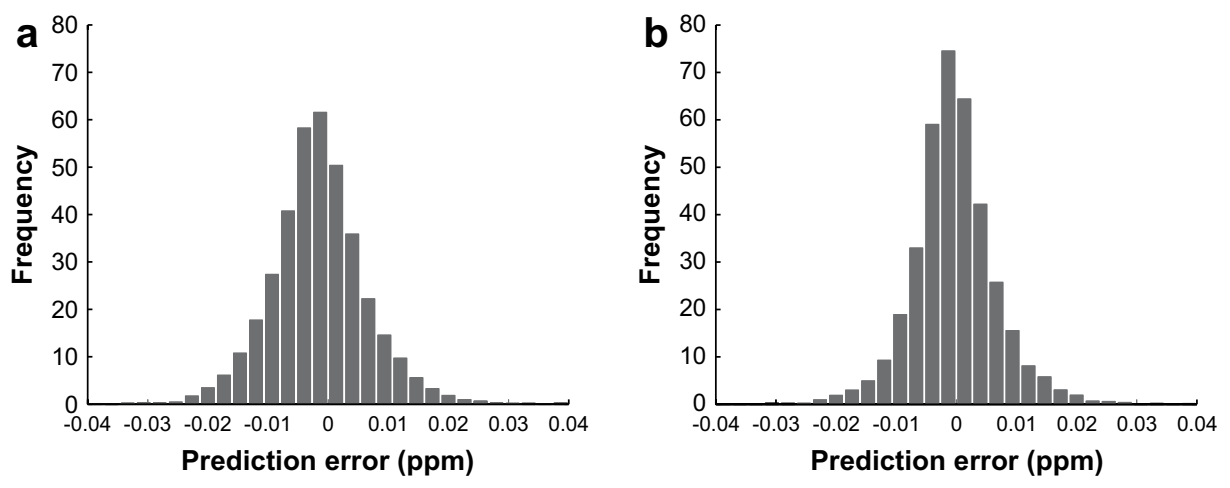

Fig. 5. $O_{3}$ mapping errors using (a) Kriging with hardened data, and (b) BME with soft data.

distinct issues. In order to facilitate the discussion, we will present and discuss them separately.

\subsection{Estimation of model components at ozone sites}

In a spatiotemporal mapping context it is useful to consider all $\widehat{S}(\boldsymbol{s}, k)$ computed from Eq. (C.1) as soft data for the non-observable $S(\boldsymbol{s}, k)$. When predicting at a space-time location where $X_{\boldsymbol{p}}$ is available, this allows us to split $X_{\boldsymbol{p}}$ according to Eq. (3).

Let us define $\boldsymbol{s}$ as one of the 262 sites locations, where we seek estimates of $X_{\boldsymbol{p}}$ and $\varepsilon_{\boldsymbol{p}}$. Since we assumed that $S_{\boldsymbol{p}} \perp \varepsilon_{\boldsymbol{p}}$, the $\mathrm{O}_{3}$ estimation is a two-steps procedure: first, an estimate $S_{\boldsymbol{p}}^{p}$ is sought based on the soft data computed by Eq. (C.1) at surrounding sites and (possibly but not necessarily) at site $\boldsymbol{s}$ itself; then, if $X_{\boldsymbol{p}}$ is available at site $\boldsymbol{s}$, an estimate of $\varepsilon_{\boldsymbol{p}}$ is given by $\widehat{\varepsilon}_{\boldsymbol{p}}=X_{\boldsymbol{p}}-S_{\boldsymbol{p}}^{p}$.

Using this procedure, it is possible to split any of the (sometimes very fragmentary) time-series of measurements as the sum of the estimated $S_{\boldsymbol{p}}^{p}$ and $\widehat{\varepsilon}_{\boldsymbol{p}}$. Corresponding results are illustrated in Fig. 3 for a few sites. It is worth remarking that even for time-series with plenty of missing values, this method allows us to obtain $S_{p}^{p}$ at any $t$, along with $\widehat{\varepsilon}_{\boldsymbol{p}}$ if $X_{\boldsymbol{p}}$ is available too.

\subsection{Mapping of the seasonal component}

Using as soft data all $\widehat{S}(\boldsymbol{s}, k)$ values computed by Eq. (C.1) and using a regular space-time grid, maps of both the seasonal $S_{\boldsymbol{p}}$ and fluctuation $\varepsilon_{\boldsymbol{p}}$ components can be generated. Fig. 4 shows the results of BME mapping of $S_{\boldsymbol{p}}$ over a spatiotemporal grid with spatial node spacing of $2 e 4 \mathrm{~m}$ (both along the North-South and West-East directions) and a temporal node spacing of 2 months. These maps clearly exhibit a spatially and temporally consistent pattern of $\mathrm{O}_{3}$ concentrations over California. The progressive change of the spatial pattern from months to months is quite obvious, with globally lowest spatial contrast (i.e. spatially more uniform $\mathrm{O}_{3}$ concentrations) from March to May, while contrast is at its maximum during winter months (December-January) and summer months (July-August). An inverse process is also clearly visible in central California, with much lower $\mathrm{O}_{3}$ concentrations during winter and much higher $\mathrm{O}_{3}$ concentrations during summer compared to other areas during the same times (in other words, during winter the central part of California always exhibits lower values than other places, whereas the opposite is true during the summer months). These areas are those characterized by the highest amplitude of the seasonal component.

Similarly, it is possible to map the fluctuation component. Since $\varepsilon_{\boldsymbol{p}}$ is not directly observable, we proceed as follows: first, estimates $\widehat{\varepsilon}_{\boldsymbol{p}}=X_{\boldsymbol{p}}-S_{\boldsymbol{p}}^{p}$ are obtained as in Section 5.1; then, based on the $\widehat{\varepsilon}_{\boldsymbol{p}}$ values, the fluctuation values can be obtained at the nodes of the spatiotemporal grid. The corresponding maps (not shown here) confirm that this component is rapidly changing over time and does not exhibit very clear spatial patterns, in agreement with the theoretical $C_{\varepsilon}(\boldsymbol{h}, \tau)$ model characterized by a short temporal correlation range and a high spatial nugget effect.

\subsection{Cross-validation assessment vs. kriging}

Beyond the $\mathrm{O}_{3}$ map generation, the spatiotemporal BME analysis can also be used to assess the adequacy of the statistical model itself using a cross-validation (i.e., leave-one-out) approach. Let us define $S_{\boldsymbol{p}}^{p}$ as the BME-estimated seasonal component at a location in which the $X_{\boldsymbol{p}}$ value was available but removed prior to spatiotemporal estimation.

We define the corresponding BME-estimated $\mathrm{O}_{3}$ residual as $\varepsilon_{\boldsymbol{p}}^{p}$, in which case we can define the cross-validation error as $X_{\boldsymbol{p}}-X_{\boldsymbol{p}}^{p}$, where $X_{\boldsymbol{p}}^{p}=S_{\boldsymbol{p}}^{p}+\varepsilon_{\boldsymbol{p}}^{p}$. A global performance indicator of $\mathrm{O}_{3}$ mapping is given by the mean square error

$\operatorname{MSE}=\frac{1}{N} \sum_{i=1}^{N}\left[X\left(\boldsymbol{s}_{i}, t\right)-X^{p}\left(\boldsymbol{s}_{i}, t\right)\right]^{2}$

Cross-validation was applied at the 162 sites that were temporarily left-out because of too many missing data (Fig. C.1). At these sites, the mapping errors are expected to be the largest and the approximations $\widehat{S}_{\boldsymbol{p}}=S_{\boldsymbol{p}}$ the worst possible. This approximation corresponds to using Kriging with "hardened" data instead of BME with soft data provided by Eq. (C.2). The results in Fig. 5 and Table 2 demonstrate that BME improves the mapping accuracy compared to Kriging, although the improvement is not dramatic here, since the soft pdf data are very narrow; this is due to the fact that $\sigma_{\varepsilon}^{2}$ is rather small compared to the total variance, so that $\sigma_{\varepsilon}^{2} / n(s, k)$ is even smaller. The $\mathrm{O}_{3}$ mapping accuracy obtained by BME is quite satisfactory, given that about $98 \%$ of the absolute mapping errors do not exceed $0.02 \mathrm{ppm}$, with a MSE that compares very well to $\sigma_{\varepsilon}^{2}=2.75 \mathrm{e}-5 \mathrm{ppm}^{2}$ (the variance of the hard-to-predict spatiotemporal component). This would also suggest that the essential part of the $\mathrm{O}_{3}$ mapping error is linked to the $\varepsilon_{\boldsymbol{p}}$ estimation error, as expected due to its limited spatiotemporal correlation. This also shows that Eq. (4), despite its simplicity, when used together with

Table 2

MSE (in $\mathrm{ppm}^{2}$ ) and probability that absolute estimation error does not exceed 0.02 ppm using Kriging and BME.

\begin{tabular}{lll}
\hline Method & MSE & $P(|E| \leq 0.02)$ \\
\hline Kriging & $6.52 \mathrm{e} 5$ & 0.979 \\
BME & $4.86 \mathrm{e} 5$ & 0.986 \\
\hline
\end{tabular}



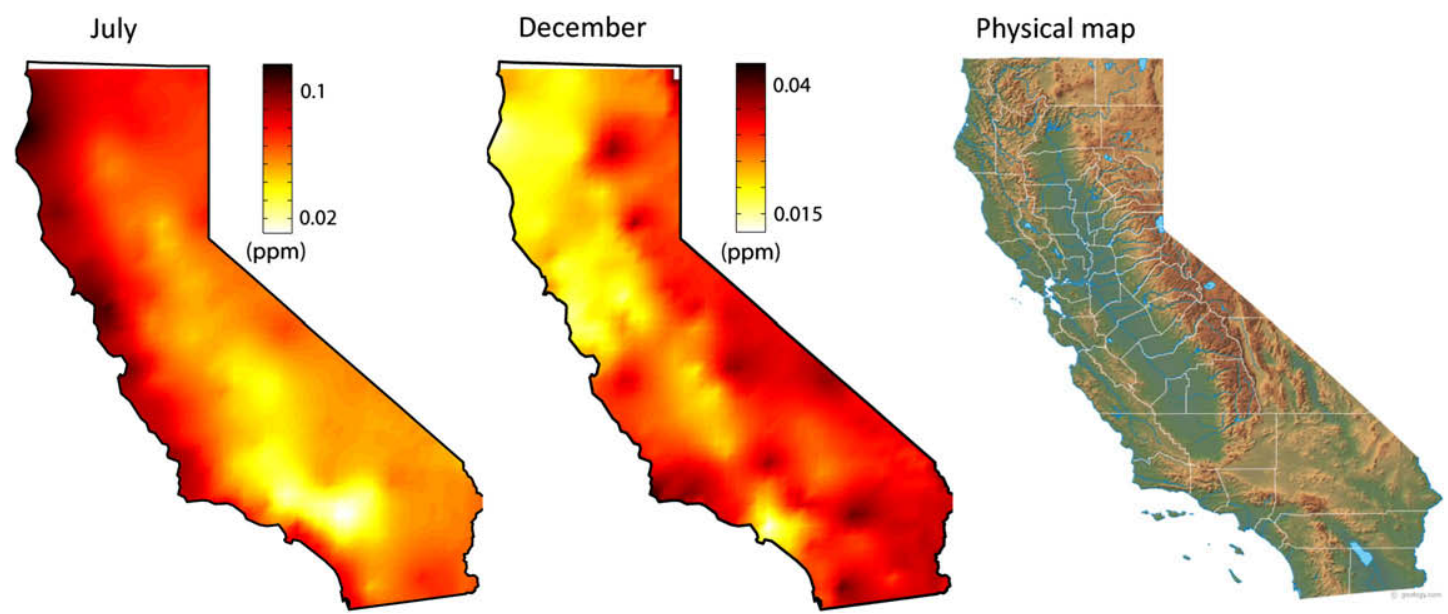

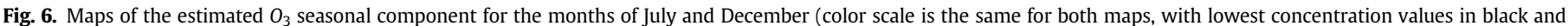

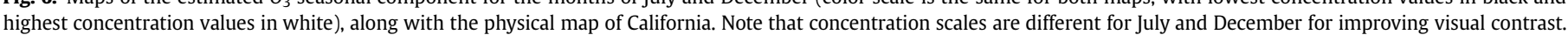

the seasonal and fluctuation covariance models and the BME method, does a good job in the spatiotemporal mapping context.

Notice that the present analysis did not account for physical laws, which reflects on the level of insight it may offer concerning the underlying $\mathrm{O}_{3}$ physical mechanisms. In other words, given the circumstances, our analysis made only limited use of the powerful BME features that allow the incorporation of important core KB in the form of physical laws and scientific theories. Nevertheless, the present analysis is a valuable tool of spatiotemporal mapping: the spatiotemporal BME framework can rigorously assimilate useful data sources that were previously unaccounted for, the generated maps offer a valuable assessment of the spatiotemporal $\mathrm{O}_{3}$ patterns that is helpful for identifying physical mechanisms and their interrelations, and the BME mapping accuracy is superior compared to other techniques commonly used by the US EPA.

\section{Discussion}

In this study, we have sought to develop a spatiotemporal prediction model for ambient ozone levels in California. This modelling represents the first stage of a multiyear investigation into the mortality effects of air pollution in the California population. We used BME analysis capable of estimating space-time levels of ozone even in the presence of missing data. The results indicate relatively little site-specific space-time interaction and considerably seasonal variation in the spatial pattern of exposure. These findings are consistent with previous understanding of ozone photochemistry (Finlayson-Pitts and Pitts, 1997). Of importance to the execution and interpretation of epidemiological studies, though, are the subtle changes to ordering of high vs. low exposure areas, with the more extreme seasonal contrasts occurring in the Central Valley. As shown in Fig. 1 a tendency exists for lower amplitudes along the coast and higher amplitudes in the south central part of the state. Insights from the spatiotemporal models indicate that coastal areas are likely to be much lower overall and more consistent through the year, while the Central Valley and inland areas of Southern California tend to demonstrate both the highest levels and highest seasonal variation. The maps in Fig. $6 \mathrm{a}$ and $\mathrm{b}$ appear to relate closely to coastal and mountain features shown in Fig. $6 c$ as protective against ozone exposure, while stagnant inland areas appear to have higher exposures. The BME model can also introduce information on other physical and land use variables to inform the interpolation. In future research, we plan to test altitude, wind, general atmospheric circulation, temperatures, land use, population density and other relevant variables to determine whether explicit inclusion improves the interpolation prediction.

\section{Conclusions}

Tropospheric Ozone is a complex, photochemical pollutant that has increased globally in concentration since the 19th century, largely as a result of increased anthropocentric emissions of nitrogen oxides and volatile organic compounds emitted from combustion and other industrial processes. Recent studies suggest that ozone will become worse over the next century partly due to global climate change (e.g., Zeng et al., 2008). Such increases may have important implications for public health because they may lengthen the ozone season in many places and increase levels overall. Because ozone is associated with a myriad of cardiorespiratory health effects, increases in the levels and the duration of the hot-season weather may exert large impacts on human populations globally. In this context, understanding the spatial extent and temporal variation in ozone exposure takes on new relevance. We have used spatiotemporal BME analysis to demonstrate their utility for understanding the complex composite space-time patterns of exposure. These assessments will yield useful information for health effects assessments and will be used to determine the sensitivity of alternative exposure estimators. The space-time assessments will also be used to assess whether critical exposure windows influence potential effects of ozone on mortality, while utilizing maximal available data. Thus, the framework presented here may enhance understanding of a pervasive pollutant that could affect population health globally.

\section{Acknowledgement}

The research was supported by a grant from the California Air Resources Board (Grant No. 55245A).

\section{Appendix A}

Below we used a technique that applies seasonal differentiation and filtering of the $S_{p}$ component, thus focusing on the fluctuation component $\varepsilon_{\boldsymbol{p}}$. This space/time technique is a particular case of the generalized S/TRF theory (Christakos, 1992; Christakos and Hristopulos, 1998). Let us define a new $\mathrm{S} / \mathrm{TRF} Y_{\boldsymbol{p}}=Y(\boldsymbol{s}, t)$ as the $1 \mathrm{st}$-order temporal increment of the original $\mathrm{O}_{3}$ field, 


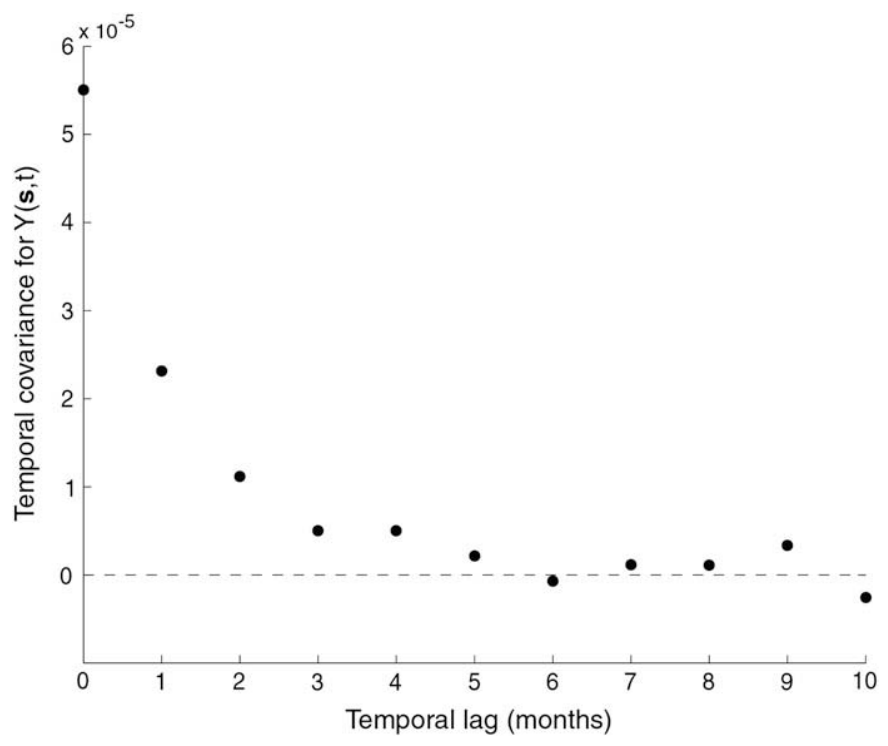

Fig. A.1. Estimated temporal covariance function $C_{Y}(\mathbf{0}, \tau)$.

$Y(\boldsymbol{s}, t)=X(\boldsymbol{s}, t)-X(\mathbf{s}, t-12)$

In view of Eqs. (3) and (4), the $Y_{\boldsymbol{p}}$ is spatially homogeneous/ temporally stationary with mean and covariance

$$
\left.\begin{array}{c}
\overline{Y(\boldsymbol{s}, t)}=\overline{\varepsilon(\boldsymbol{s}, t)}-\overline{\varepsilon(\boldsymbol{s}, t-12)}=0 \\
C_{Y}(\boldsymbol{h}, \tau)=\operatorname{Cov}[Y(\boldsymbol{s}, t), Y(\boldsymbol{s}+\boldsymbol{h}, t+\tau)]
\end{array}\right\}
$$

Furthermore, Eqs. (A.1) and (A.2) yield

$$
\begin{aligned}
C_{Y}(\boldsymbol{h}, \tau) & =\overline{[X(\boldsymbol{s}, t)-X(\boldsymbol{s}, t-12)][X(\boldsymbol{s}+\boldsymbol{h}, t+\tau)-X(\boldsymbol{s}+\boldsymbol{h}, t+\tau-12)]} \\
& =\overline{[\varepsilon(\boldsymbol{s}, t)-\varepsilon(\boldsymbol{s}, t-12)][\varepsilon(\boldsymbol{s}+\boldsymbol{h}, t+\tau)-\varepsilon(\boldsymbol{s}+\boldsymbol{h}, t+\tau-12)]} \\
& =2 C_{\varepsilon}(\boldsymbol{h}, \tau)-C_{\varepsilon}(\boldsymbol{h}, \tau-12)-C_{\varepsilon}(\boldsymbol{h}, \tau+12)
\end{aligned}
$$

The purely spatial and purely temporal covariances are given by, respectively,

$$
\begin{aligned}
& C_{Y}(\boldsymbol{h}, 0)=2 C_{\varepsilon}(\boldsymbol{h}, 0)-C_{\varepsilon}(\boldsymbol{h},-12)-C_{\varepsilon}(\boldsymbol{h}, 12) \\
& C_{Y}(\mathbf{0}, \tau)=2 C_{\varepsilon}(\mathbf{0}, \tau)-C_{\varepsilon}(\mathbf{0}, \tau-12)-C_{\varepsilon}(\mathbf{0}, \tau+12)
\end{aligned}
$$

These spatial (temporal) covariance functions are symmetric with respect to $\boldsymbol{h}(\tau)$. Obtaining $C_{\varepsilon}(\boldsymbol{h}, \tau)$ from $C_{Y}(\boldsymbol{h}, \tau)$ would require inverting Eq. (A.3), an affair that typically involves first some parametric model $C_{\varepsilon}(\boldsymbol{h}, \tau ; \theta)$ and then looking for the $\theta$ 's that maximize, say, the likelihood of the $Y(s, t)$ with respect to this model. However, as is shown in Appendix B, under certain reasonable conditions the following approximations are valid

$$
\begin{array}{ll}
C_{Y}(\boldsymbol{h}, \tau) \cong 2 C_{\varepsilon}(\boldsymbol{h}, \tau) & \text { (a) } \\
\gamma_{Y}(\boldsymbol{h}, \tau) \cong 2 \gamma_{\varepsilon}(\boldsymbol{h}, \tau) \quad \text { (b) }
\end{array}
$$

where $\gamma(\cdot)$ denotes the variogram. Then, a non-parametric estimation of $C_{Y}(\boldsymbol{h}, 0)$ and $C_{Y}(\mathbf{0}, \tau)$ is possible, in which case the relations

$$
\begin{aligned}
& C_{\varepsilon}(\boldsymbol{h}, 0) \cong \frac{1}{2} C_{Y}(\boldsymbol{h}, 0) \quad(\mathbf{a}) \\
& C_{\varepsilon}(\mathbf{0}, \tau) \cong \frac{1}{2} C_{Y}(\mathbf{0}, \tau) \quad \text { (b) }
\end{aligned}
$$

can be used afterwards. From Fig. A.1 and Eq. (5) it can be verified that the estimated covariance values are close to 0 for $\tau=3$ months, so that Eq. (A.6) provide sound estimates $\widehat{C}_{\varepsilon}(\boldsymbol{h}, 0) \cong 1 / 2 \widehat{C}_{Y}(\boldsymbol{h}, 0)$ and $\widehat{C}_{\varepsilon}(\mathbf{0}, \tau) \cong 1 / 2 \widehat{C}_{Y}(\mathbf{0}, \tau)$.

The exponential model offers a good fit to the temporal variogram (Fig. A.2a). The spatial variogram has a significant nugget effect (about $1 / 2$ the total variance); whereas the remaining part could be associated with a long-range spatial structure (Fig. A.2b) which, in practice can be also approximated by an exponential model with a very long-range. Thus, the space-time separable covariance model of $\varepsilon(\boldsymbol{s}, t)$ is given in Eq. (5).

\section{Appendix B}

Under certain conditions the $C_{\varepsilon}(\boldsymbol{h}, \tau)$ can be derived from $C_{Y}(\boldsymbol{h}, \tau)$, as follows. Let $C_{\varepsilon}(\boldsymbol{h}, \tau)$ be monotonically decreasing with $|\tau|$ and approaches 0 beyond a time-lag $\tau_{0}: 0<\tau_{0}<12$. Then from Eq. (A.4) we get

$$
\begin{aligned}
& C_{\varepsilon}(\mathbf{0}, \tau+12) \cong 0, \quad \tau \geq 0 \quad(\mathrm{a}) \\
& C_{\varepsilon}(\mathbf{0}, \tau-12) \cong 0, \quad|\tau-12| \geq \tau_{0}
\end{aligned}
$$

For a positive-valued and monotonically decreasing $C_{\varepsilon}(\boldsymbol{h}, \tau)$ with both $|\boldsymbol{h}|$ and $|\tau|$ (a rather typical behavior), we obtain $0<C_{\varepsilon}(\boldsymbol{h},-12)$ $\leq C_{\varepsilon}(\mathbf{0},-12) \cong 0$ and $0<C_{\varepsilon}(\boldsymbol{h}, 12) \leq C_{\varepsilon}(\mathbf{0}, 12) \cong 0$; i.e., $C_{\varepsilon}(\boldsymbol{h},-12)$ $=C_{\varepsilon}(\boldsymbol{h}, 12) \cong 0$. Combining the above results, we find
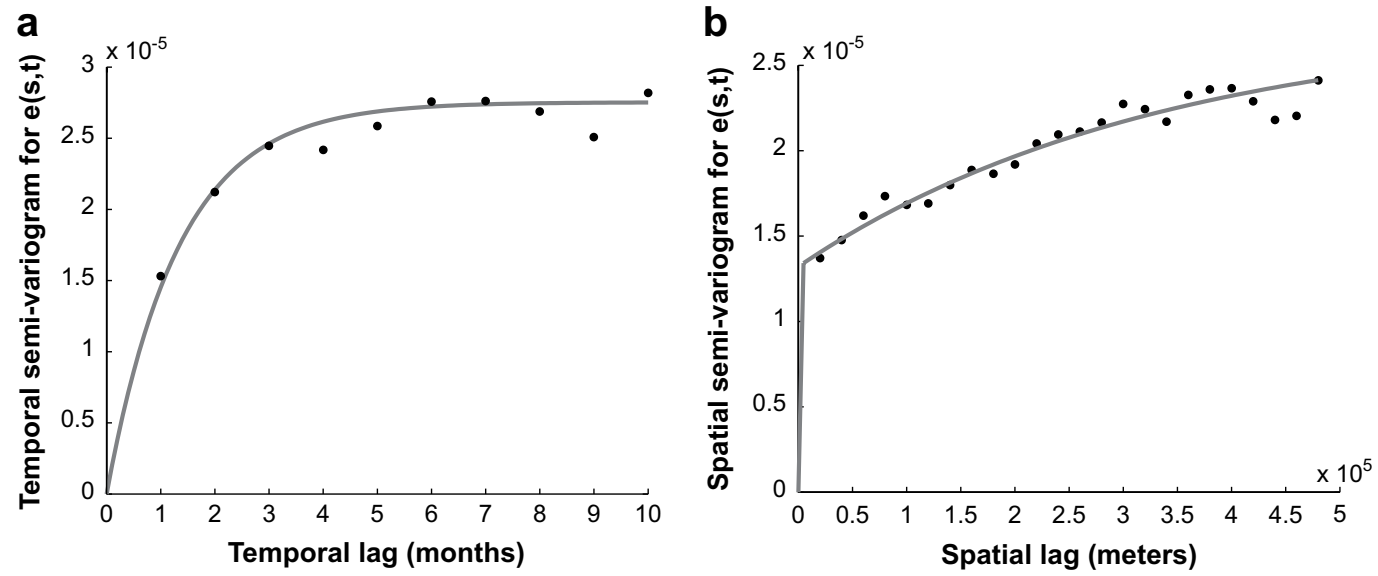

Fig. A.2. Estimated and fitted (a) temporal variogram $\gamma_{\varepsilon}(\boldsymbol{h}, 0)$ and $(b)$ spatial variogram $\gamma_{\varepsilon}(\mathbf{0}, \tau)$. 


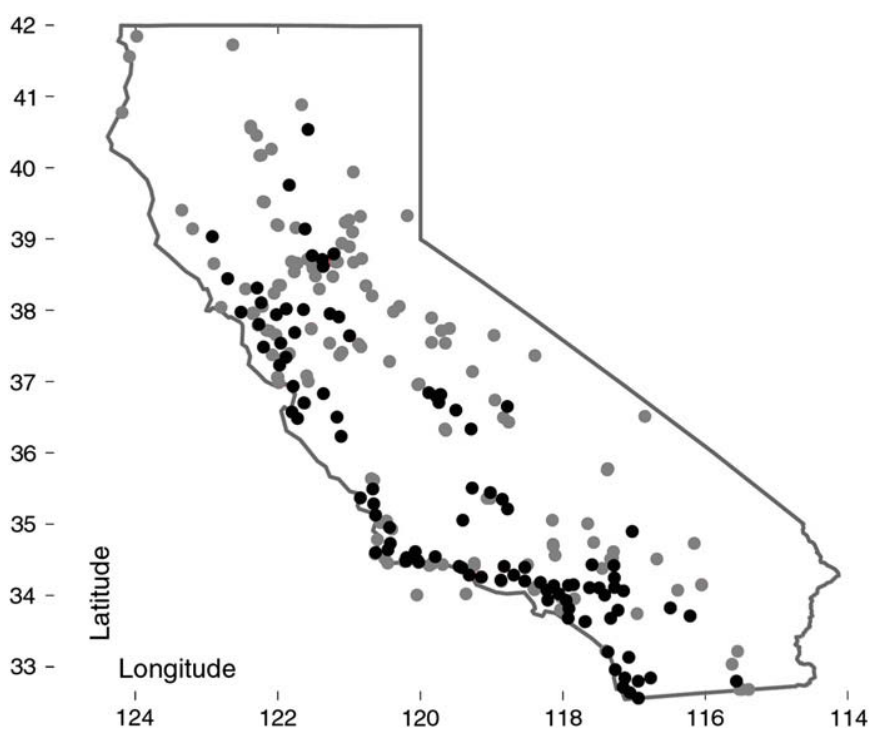

Fig. C.1. Location of the 100 sites with less than 10 available data (in gray); and 162 sites with at least 10 available data (in black) for each of the 12 months of the year, during the years 1988-2002.

Eq. (A.5a). In a similar way we derive Eq. (A.5b). Thus, the above conditions allow a non-parametric estimation of $C_{Y}(\boldsymbol{h}, 0)$ and $C_{Y}(\mathbf{0}, \tau)$.

\section{Appendix C}

For inference purposes, if we assume that $S(\boldsymbol{s}, t) \perp \varepsilon(\boldsymbol{s}, t)$, obvious estimators of $S(\boldsymbol{s}, k)$ are

$$
\left.\begin{array}{c}
\widehat{S}(\boldsymbol{s}, 1)=\frac{1}{n(\mathbf{s}, 1)} \sum_{j} X(\mathbf{s}, 1+12 j) \\
\vdots \\
\widehat{S}(\boldsymbol{s}, 12)=\frac{1}{n(\boldsymbol{s}, 12)} \sum_{j} X(\mathbf{s}, 12+12 j)
\end{array}\right\}
$$

for all locations $\boldsymbol{s}(j=0,1, \ldots, 12)$; the $n(\cdot)$ refers to the number of data that vary with respect to $s$ and $k$ due to missing values. Basically, Eq. (C.1) is equivalent to averaging values belonging to the same month of a year over all years (i.e., all January values, all February values etc.). From the central limit theorem and since Eq. (5) implies $C_{Y}(\boldsymbol{h}, 12 j) \cong 0 \quad(j \neq 0)$, a reasonable distributional hypothesis is

$\widehat{S}(\boldsymbol{s}, k) \sim N\left(S(\boldsymbol{s}, k), \frac{\sigma_{\varepsilon}^{2}}{n(\boldsymbol{s}, k)}\right)$

for all $k$. Moreover, estimates of $C_{S}(\boldsymbol{h}, k)$ and $\gamma_{S}(\boldsymbol{h}, k)$ can be computed by substituting $S(\boldsymbol{s}, k)$ with $\widehat{S}(\boldsymbol{s}, k)$ as long as $\sigma_{\varepsilon}^{2} / n(\boldsymbol{s}, k)<<\sigma_{S, k}^{2}=C_{S}(\mathbf{0}, k)$. The last hypothesis amounts to replacing the true $S(\boldsymbol{s}, k)$ by their estimates $\widehat{S}(\boldsymbol{s}, k)$ for the purpose of estimating the covariances and variograms. This does not have a noticeable impact on the estimation of $C_{S}(\boldsymbol{h}, k)$ and $\gamma_{S}(\boldsymbol{h}, k)$, as long as the variability of these estimates is small compared to the spatial variability of the $S(\boldsymbol{s}, k)$. It is worth noting that the residuals could be also directly estimated, since from Eq. (3) we have $\widehat{\varepsilon}_{\boldsymbol{p}}=X_{\boldsymbol{p}}-\widehat{S}_{\boldsymbol{p}} \cong \varepsilon_{\boldsymbol{p}}$. This would provide another way of estimating $C_{\varepsilon}(\boldsymbol{h}, \tau)$ directly from the $\widehat{\varepsilon}_{\boldsymbol{p}}$, instead of using the seasonal filtering of the $X_{p}$, as was done in Appendix A.

In order to keep $\sigma_{\varepsilon}^{2} / n(\boldsymbol{s}, k)$ as low as possible, while at the same time allowing enough data for a reliable estimation of the seasonal covariance $C_{S}(\boldsymbol{h}, k)$, Eq. (C.1) was only used in cases that it was valid that $n(s, k) \geq 10$ for all $k$; i.e., when $\operatorname{Var}[\widehat{S}(\boldsymbol{s}, k)]=\sigma_{\varepsilon}^{2} / 10=2.75 \mathrm{e}-6 \mathrm{ppm}^{2}$. This corresponds to 100
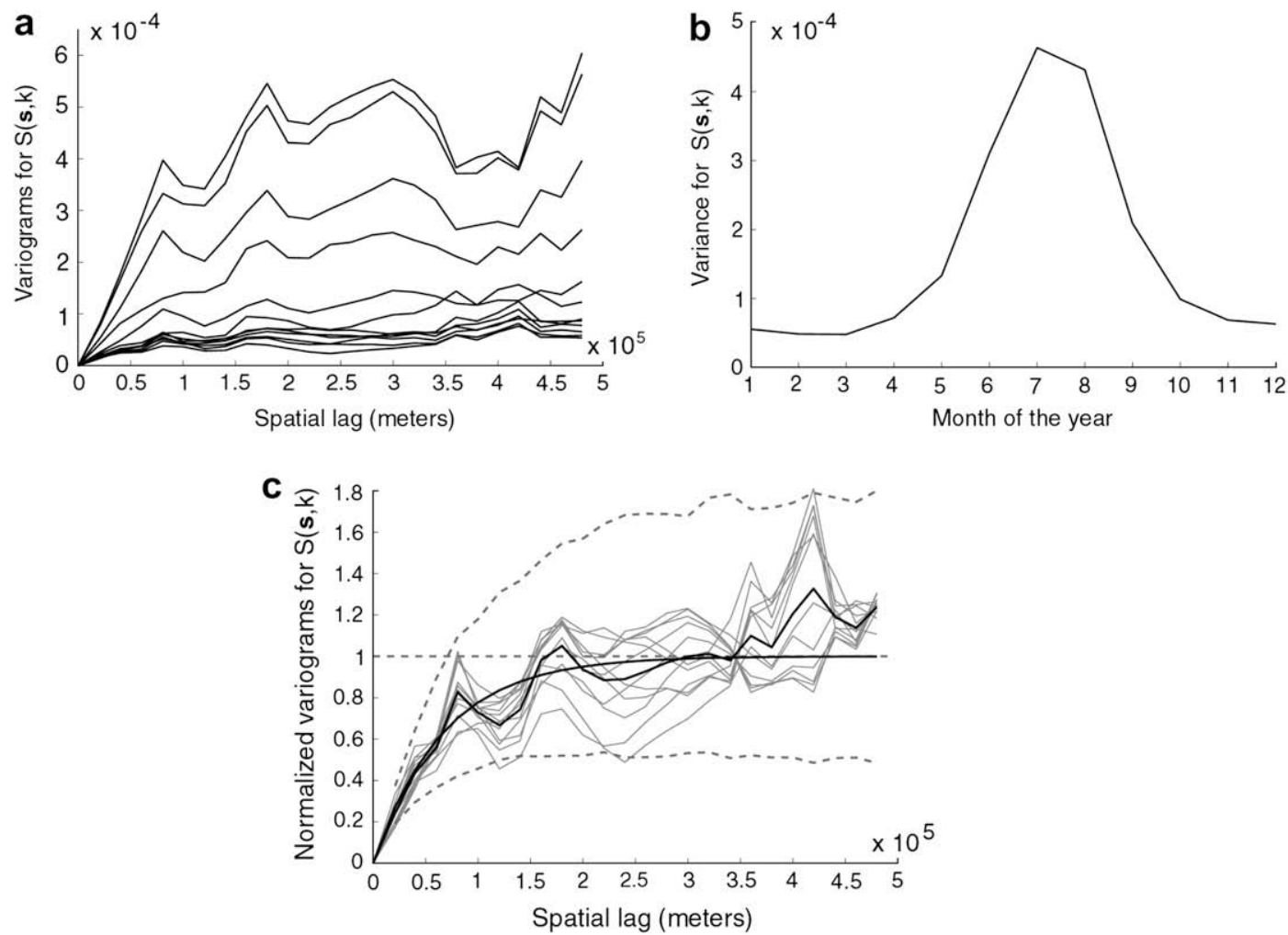

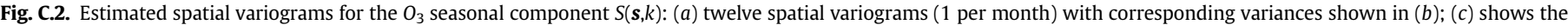

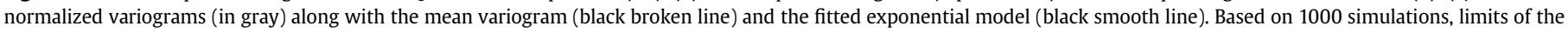
estimated 95\% confidence interval for the exponential model are shown as dashed gray lines. 
out of the 262 original sites (Fig. C.1). For these 100 sites, it can be verified from Fig. C.2 that, if we use $\widehat{S}(\boldsymbol{s}, k)$ instead of $S(\boldsymbol{s}, k)$ to estimate $C_{S}(\boldsymbol{h}, k)$ and $\gamma_{S}(\boldsymbol{h}, k)$, the variance of the $\widehat{S}(\boldsymbol{s}, k)$ is at least one order of magnitude lower than the spatial variability of $S(s, k)$ during the winter months (which are months exhibiting the lower variance over space), and about two orders of magnitude lower during the summer months (which are months exhibiting the highest variance over space). This result was already expected from Table 1 , which emphasized the limited contribution of $\sigma_{\varepsilon}^{2}$ to the total $\mathrm{O}_{3}$ variance.

Although the spatial variance of the seasonal $\mathrm{O}_{3}$ component is changing over time, a simple normalization of the various $\widehat{\gamma}_{S}(\boldsymbol{h}, k)$ 's shows that they compare relatively well and they could be fitted by the same exponential model (Fig C.2c). Based on 1000 simulations using this model, it is possible to draw an estimated $95 \%$ confidence interval for the variogram estimator conditionally to the model. For this, the fitted variogram model was used to generate a set of 1000 independent realizations at similar space-time locations than those that were used for computing the variogram estimates. The corresponding 1000 variogram estimates were then computed and the results were used for deriving a 95\% estimated confidence interval (dashed lines in Fig C.2c). As this confidence interval includes all $\widehat{\gamma}_{S}(\boldsymbol{h}, k)$, there is no reason to reject the hypothesis that it is acceptable to use the same exponential model for all months. In other words, the variability of an estimated variogram based on this exponential model is at least equal to (if not greater than) the variability observed among the $\widehat{\gamma}_{S}(\boldsymbol{h}, k)$ 's. Based on these results, the finally selected covariance model for the $\mathrm{O}_{3}$ seasonal component $S(s, k)$ is given by Eq. (6).

\section{References}

Bogaert, P., Christakos, G., 1997a. Stochastic analysis of solute content measurements using a regressive space-time model. Stoch. Hydrol. and Hydraul. 11 (4), 267-296.

Bogaert, P., Christakos, G., 1997b. Spatiotemporal analysis and processing of thermometric data over Belgium. J. Geophys. Res. - Atmospheres 102 (D22), 2583125846.

Carroll, R.J., Chen, R., George, E.I., Li, T.H., Newton, H.J., Schmiedliche, H., Wang, N., 1997. Ozone exposure and population density in Harris County, Texas. J. Am. Stat. Assoc. 92, 392-404.

Chang, J.S., Brost, R.A., Isaksen, I.S.A., Madronich, S., Middleton, P., Stockwell, W.R., Walcek, C.J., 1987. A three-dimensional Eulerian acid deposition model: physical concepts and formulation. J. Geophys. Res. 92, 14681-14700.

Chen, C.-C., Wu, K.-Y., Chang, M.J.W., 2004. A statistical assessment on the stochastic relationship between biomarker concentrations and environmental exposures. Stoch. Env. Res. and Risk A. 18, 377-385.

Chen, T.-M., Gokhale, J., Shofer, S., Kuschner, W.G., 2007. Outdoor air pollution: ozone health effects. Am. J. Med. Sci. 333 (4), 244-248.

Christakos, G., 1992. Random Field Models in Earth Sciences. Academic Press, Inc., San Diego, CA.

Christakos, G., Hristopulos, D.T., 1998. Spatiotemporal Environmental Health Modelling: a Tractatus Stochasticus. Kluwer Acad. Publ., Boston, MA.

Christakos, G., Vyas, V., 1998. A composite spatiotemporal study of ozone distribution over Eastern United States. Atmos. Environ. 32 (16), 2845-2857.

Christakos, G., Kolovos, A., 1999. A study of the spatiotemporal health impacts of ozone exposure. J. Expo. Anal. Environ. Epidemiol. 9 (4), 322-335.
Christakos, G., 2000. Modern Spatiotemporal Geostatistics. Oxford Univ. Press, New York, NY.

Christakos, G., Bogaert, P., Serre, M.L., 2002. Temporal GIS. Springer-Verlag, New York, NY.

Christakos, G., Kolovos, A., Serre, M.L., Vukovich, F., 2004. Total ozone mapping by integrating data bases from remote sensing instruments and empirical models. IEEE T. Geosci. and Remote 42 (5), 991-1008.

Christakos, G., 2008. Bayesian maximum entropy. In: Kanevski, M. (Ed.), Advanced Mapping of Environmental Data: Geostatistics, Machine Learning, and Bayesian Maximum Entropy-Chapter 6. J. Wiley \& Sons, New York, NY, pp. 247-306.

Clark, T.L., 1980. Annual anthropogenic pollutant emissions in the US and the southern Canada east of the Rocky Mountains. Atmos. Environ. 14, 961-970.

Diem, J.E., 2000. Comparisons of weekday-weekend ozone: importance of biogenic volatile organic compound emissions in the semi-arid southwest USA. Atmos. Environ. 34, 3445-3451.

Finlayson-Pitts, B.J., Pitts Jr., J.N., 1997. Tropospheric air pollution: ozone, airborne toxics, polycyclic aromatic hydrocarbons, and particles. Science 276, 1045-1052.

Huang, H., Martinez, F., Mateu, J., Montes, F., 2007. Model comparison and selection for stationary space-time models. Comput. Stat. Data An. 51 (9), 4577-4596.

Jacobson, M.Z., 1999. Fundamentals of Atmospheric Modeling. Cambridge Univ. Press, New York, NY.

Jerrett, M., Burnett, R.T., Ma, R., Pope III, C.A., Krewski, D., Newbold, K.B. Thurston, G., Shi, Y., Finkelstein, N., Calle, E.E., Thun, M.J., 2005. Spatial analysis of air pollution and mortality in Los Angeles. Epidemiology 16 (6), 727-736.

Koike, E., Kobayashi, T., 2004. Ozone exposure enhances antigen-presenting activity of interstitial lung cells in rats. Toxicology 196, 217-227.

Kolovos, A., Yu, H.-L., Christakos, G., 2006. SEKS-GUI v.0.6 User Manual. Dept. of Geography, San Diego State University, San Diego, CA.

Logan, J.A., 1989. Ozone in rural areas of the US. J. Geophys. Res. 94 (D6), 8511-8532.

Mudway, I.S., Kelly, F.J., 2004. An investigation of inhaled ozone dose and the magnitude of airway inflammation in healthy adults. Am. J. Respir. Crit. Care. Med. 169, 1089-1095.

Mutshinda, C.M., Antai, I., O’Hara, R.B., 2007. A probabilistic approach to exposure risk assessment. Stoch. Env. Res. Risk A. 22, 441-449.

Ostro, B.D., Tran, H., Levy, J.I., 2006. The health benefits of reduced tropospheric ozone in California. J Air Waste Manag. Assoc. 56 (7), 1007-1021.

Schneider, Ch., Kessler, Ch., Moussiopoulos, N., 1997. Influence of emission input data on ozone level predictions for the upper Rhine Valley. Atmos. Environ. 31, 3187-3205.

Solomon, P., Cowling, E., Hidy, G., Furiness, C., 2000. Comparison of scientific findings from major ozone field studies in North America and Europe. Atmos. Environ. 34, 1885-1920.

Sram, R.J., Binkova, B., Dejmek, J., Bobak, M., 2005. Ambient air pollution and pregnancy outcomes: a review of the literature. Environ. Health Perspect. 113, 375-382.

Tao, Z., Larson, S.M., Williams, A., Caugheyc, M., Wuebbles, D.J., 2004. Sensitivity of regional ozone concentrations to temporal distribution of emissions. Atmos. Environ. 38, 6279-6285.

Tao, Z., Larson, S.M., Williams, A., Caugheyc, M., Wuebbles, D.J., 2005. Area, mobile, and point source contributions to ground level ozone: a summer simulation across the continental USA. Atmos. Environ. 39, 1869-1877.

Taylor, A.C., 1993. Using objective and subjective information to develop distributions for probabilistic exposure assessment. J. Expo. Anal. Environ. Epidemiol. 3 (3), 285-298.

Thurston, G.D., Ito, K., 2001. Epidemiological studies of acute ozone exposures and mortality. J. Expo. Anal. Environ. Epidemiol. 11, 286-294.

Trainer, M., Williams, E.J., Parrish, D.D., Buhr, M.P., Allwine, E.J., Westberg, H.H. Fehsenfeld, F.C., Liu, C.S., 1987. Models and observations of the impact of natural hydrocarbons on rural ozone. Nature 329, 705-707.

Yu, H.-L., Kolovos, A., Christakos, G., Chen, J.-C., Warmerdam, S., Dev, B., 2007. Interactive spatiotemporal modelling of health systems: the SEKS-GUI framework. Stoch. Env. Res. Risk A. 21 (5), 555-572.

Yu H.-L., Chen J.-C., Christakos G., Jerrett M. 2008. Estimation of residential exposure to ambient $\mathrm{PM}_{10}$ and ozone at multiple time-scales. Environ. Health Perspect. doi:10.1289/ehp.0800089. [online 15 December 2008].

Zeng, G., Pyle, J.A., Young, P.J., 2008. Impact of climate change on tropospheric ozone and its global budgets. Atmos. Chem. Phys. 8, 369-387. 